\title{
More possibilities from Rivastigmine to new potential agents on treating Alzheimer's disease: In silico study
}

\author{
Zhi-Gang Sun ${ }^{1,2 *}$, Qing Yuan ${ }^{1}$ and Yu-Shun Yang ${ }^{1}$ \\ ${ }^{1}$ School of Life Sciences, Nanjing University, Nanjing 210023, PR China \\ ${ }^{2}$ Central Laboratory, Linyi Central Hospital, Linyi 276400, Shandong, PR China
}

\begin{abstract}
Alzheimer's disease (AD) is one of the most hazardous and frequent chronic neurodegenerative disease around the world. Though several hypotheses have been raised, four in five of the FDA certified drugs for treating AD were acetylcholinesterase inhibitors. This work raised more possibilities on substituent-positions and conformations from the most potent AChEI Rivastigmine. The molecular docking indicated the probable advantage of the para-position and the S-conformation. The key interactions with residues including TYR121 and HIS440 were recognized in the visualized binding patterns. The information raised here was valuable for future modifications and designs of AChEIs.
\end{abstract}

\section{Introduction}

Along with the development of the modern world, advanced medicinal and therapeutic conditions have encouraged longevity to be the common sense [1]. Concomitantly, the usual defined diseases for elder population, have forced the researchers to face the fact that they are occurring with higher percentages and stretching their claws to younger people [2,3]. Among them, Alzheimer's disease (AD) is of course one of the most hazardous and frequent, which ruined the capabilities of remembering recent things, organizing language, distinguishing orientations and controlling mood [4,5]. AD is a widely known chronic neurodegenerative disease and it caused a majority of dementia cases (over 60\%) [6,7]. On the contrary, the cause of AD is still poorly revealed. Since the major phenomena are amyloid beta (A $\beta)$ deposits, tau protein abnormalities and neuronal death, several hypotheses have been raised accordingly [8-10]: 1) Genetic cause, mainly the mutations in genes encoding amyloid precursor protein (APP) and presenilins 1 and 2 [11];2) Cholinergic hypothesis, the most typical one with the most currently available drugs based [12-14]; 3) Amyloid hypothesis, the currently leading opinion in this field but the two developed vaccines Bapineuzumab and Solanezumab both failed the Phase III clinical trials [15-18]; 4) Tau hypothesis, the most accepted target following the amyloid with potential agents on the promotion of Tau's decomposition and the inhibition of its accumulation or phosphorylation [19-21]; 5) Other hypotheses including inflammation, aluminium, smoking and gum diseases [22-25]. In spite of the antiamyloid vaccine's failures, the very recent study also questioned the rationality of inhibition Tau because its multiple functions [26]. Thus, acetylcholinesterase inhibitors (AChEI) are still the down-to-earth choices of the patients of $\mathrm{AD}$.

Despite Menantine Hydrochloride, other four FDA certified drugs (Figure 1) are all AChEIs, including Tacrine, Donepezil, Galanthamine and Rivastigmine [27-30]. Tacrine lost its market because of its hepatotoxicity. Donepezil and Galanthamine are both reversible inhibitors, indicating limited treatment effect thus being recommended for the early and moderate period of $\mathrm{AD}$. Only Rivastigmine is the pseudo-irreversibility inhibitor, which can prolong the existing time of acetylcholine thus protect the conduction of neural signals. An extra inhibition site of Rivastigmine may be Butyrylcholinesterase (BuChE), another possible target of treating $\mathrm{AD}$. Developing new trails on AChEIs from Rivastigmine is a meaningful and practical approach for curing $\mathrm{AD}$ and even other neurodegenerative disease.

In this work, we extended the possibilities of Rivastigmine from two dimensions, substitution site and steric conformation. By investigating the binding patterns of extended choices into the protein structure of $\mathrm{AChE}$, we attempted to reveal one corner of the iceberg on developing deeper and further achievements in AD treatment (Figure 1).

\section{Materials and methods}

According to the basic structure of Rivastigmine, we considered the meta- (original), ortho- and para- substituent situations to develop new molecules. Meanwhile, the opposite conformations were also involved. The studied molecules were listed in Figure 2. Then their conformation optimizing process was conducted by energy minimization via CHARMm force field. In order to guarantee the final conformations, obey the rule of energy minimized, the iteration rounds were set as 5000, which was far more than the real requirement. After that, we checked the consistency of the designed ones and the optimized ones to avoid reversal.

Molecular docking simulation was performed by the CDOCKER project of Discovery Studio software (Accelrys, USA). The crystal structures of $\mathrm{AChE}$ (PDB Code: 1GQR) was selected from the RCSB

${ }^{\star}$ Correspondence to: Zhi-Gang Sun, School of Life Sciences, Nanjing University, Nanjing 210023, PR China, E-mail: zhigangsunny@nju.edu.cn

Key words: Alzheimer's disease, acetylcholinesterase inhibitors, rivastigmine, substituent position, conformation, molecular docking

Received: December 21, 2018; Accepted: January 28, 2019; Published: February 01,2019 


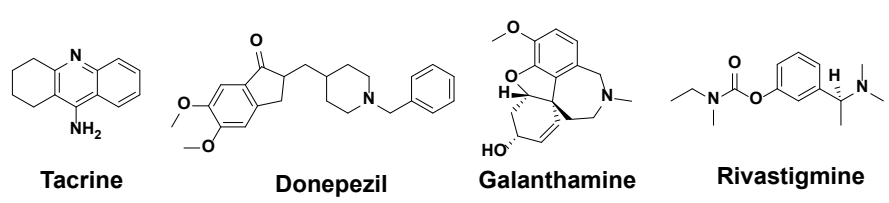

Figure 1. The four FDA certified AChEIs

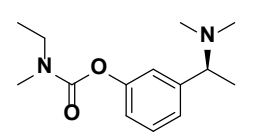

S1<smiles>CCN(C)C(=O)Oc1cccc([C@H](C)N(C)C)c1</smiles>

R1<smiles>CCN(C)C(=O)Oc1ccccc1C(C)N(C)C</smiles>

S2

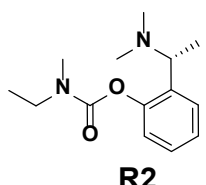

R2

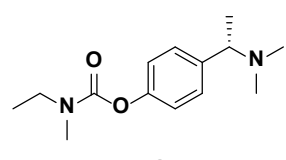

S3

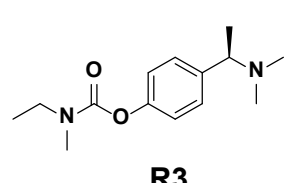

R3
Figure 2. The expansion of Rivastigmine

Protein Data Bank (http://www.rcsb.org). The protein preparation procedure followed the standard protocol. Before docking, the original ligand and water molecules were deleted from the protein crystal structure. And the polar hydrogen was added to the cleaned proteins. The binding site were defined from the same site of Rivastigmine. The binding site was checked to keep the suitable radius of the sphere (0.5$10 \mathrm{~nm}$ ) to increase the relatively reliable of the model. For a second time, CHARMm force field was used to complete the docking using the CDOCKER program with the random generated ligand conformations and a half-flexible receptor. Basic steps were as follows:

First, the ligands conformations in series are generated by high temperature molecular dynamics with various random seeds. Second, random orientations of the conformations are subsequently created by translating the center of the ligand to a specified position within the receptor active site and making a series of random rotations with calculated softened energy. Then the orientation is kept until reaching a specified limit. The process repeats before either obtaining the desired number of low-energy orientations or reaching the maximum number of the test times of bad orientations. Third, all orientations are subjected to simulated annealing molecular dynamics. The temperature is heated up to a high temperature then cooled to the target temperature. A final energy minimization of the ligand in the rigid receptor using nonsoftened potential is then conducted. Finally, the CHARMm energy (interaction energy plus ligand strain) and the interaction energy alone are figured out for each final pose. The poses are sorted according to CHARMm energy and the top scoring (most negative, thus favorable to binding) poses are restored. The whole kinase domain defined as a receptor and the site sphere is selected based on the original ligand binding location, then the original ligand is removed, and the ligands prepared are placed during the molecular docking procedure. In the simulated annealing method, the heating steps are 2000 with 700 of heating target temperature and the cooling steps are 5000 with 300 cooling target temperature. For each ligand, ten final poses are saved according to their dock score rank. The pose with the lowest energy is chosen as the top one. The pattern analysis is subsequently performed.

\section{Results and discussion}

Since the binding site was defined from the original one of Rivastigmine, we thought the original ligand Rivastigmine (S1) should be the best interacted one. The result was interesting that if simply consider the binding situations into AChE, Rivastigmine was not the best choice. As presented in Table 1, we took the minus values of the interaction energy and complex energy. Thus, the larger the values were, the lower the energy was, then the easier the binding would happen or the more stable the complex was. Though the data base was not large, there were still some hints for future researches. First, although both the substituent positions and conformations were important factors, the former seemed more essential due to larger change in steric situations. Both the $R$ and $S$ conformations of para-substituted compounds suggested better interaction with AChE. Second, when we only compared the substituent positions, we could reach the order of para- $>$ (">" meant "better than") meta- > ortho- in both conformations. This result inferred that the steric hindrance might block the acetylcholinemimic function of AChEIs, thus retaining a relative free terminal would be necessary in designing such inhibitors. Third, if we only compare the conformations, the obvious fact was $S->R$ - in all positions. This might be the real desire of AChE binding. Subsequently, with all the hints involved, could the top hits be more potential than Rivastigmine? The answer was positive but not definite. For the two candidates S3 and R3, they both had some doubts yet. For R3, an obvious problem was that its complex energy was higher than Rivastigmine. That means, even it could realize the easier interaction with the target enzyme, it would also separate in a shorter period. Since AChEIs usually attempted to stick with AChE longer to allow the passing of acetylcholine, R3 seemed conflict with this basic demand. For S3, it was closer to Rivastigmine and provided better parameters in both interaction energy and complex energy. Even all the conditions seemed that $\mathrm{S} 3$ could be a more potent hit, further investigations should be conducted step by step to verify its real performances. Because easier interaction and longer binding period might cause other problems such as neurological disorders or a different type of nerve disease. Anyway, the good news was that para-substituted trails could be taken into consideration in future modifications and designs to enrich the backbones of AChEIs.

To visualize the binding patterns of the studied compounds, we presented the $2 \mathrm{D}$ maps in Figure 3. Though Rivastigmine was presupposed in other works to decompose from ester group, we took the original status of the studied compounds directly into the active site of AChE to evaluate their initial binding situations. As shown in Figure 3, a major difference between S-conformations and R-conformations was the categories of key interactions. The S-conformations preferred direct hydrogen bonds and sometimes indirect $\pi-\sigma$ interactions, whereas the R-conformations all employed $\pi-\pi$ or $\pi$-cation interactions. According to the reports, if Rivastigmine decomposed, the binding would be weakened to two $\pi-\pi$ interactions with TRP84 and PHE330 respectively. Thus, the original status here seemed to indicate better interactions. By retaining the same hydrogen bond with TYR121 with the same pattern and introducing more hydrogen bond with HIS440, S3 and R3 suggested more potential binding pattern than Rivastigmine. The failure of the other three candidates could also be inferred by the binding patterns. R1 lost all potential hydrogen bonds. S2 and $\mathrm{R} 2$ seemed to be rotated or reversed in the binding site as their key interactions did not appear at the same positions. The above results were cheerful because the recommended compound S3 was still in similar pattern with Rivastigmine, which enhanced the reliability of

Table 1. The interaction energy and complex energy of the compounds into AChE

\begin{tabular}{|c|c|c|c|c|c|}
\hline Compound & $\begin{array}{c}\text {-Interaction } \\
\text { Energy } \\
\text { (kcal/mol) }\end{array}$ & $\begin{array}{c}- \text { Complex } \\
\text { Energy } \\
\text { (kcal/mol) }\end{array}$ & Compound & $\begin{array}{c}\text {-Interaction } \\
\text { Energy } \\
\text { (kcal/mol) }\end{array}$ & $\begin{array}{c}\text {-Complex } \\
\text { Energy } \\
\text { (kcal/mol) }\end{array}$ \\
\hline S1 & 39.8686 & 33.4468 & R1 & 39.6957 & 31.7529 \\
\hline S2 & 39.7678 & 32.3533 & R2 & 39.6621 & 33.0382 \\
\hline S3 & 41.3823 & 34.3766 & R3 & 41.0413 & 32.0519 \\
\hline
\end{tabular}


S1

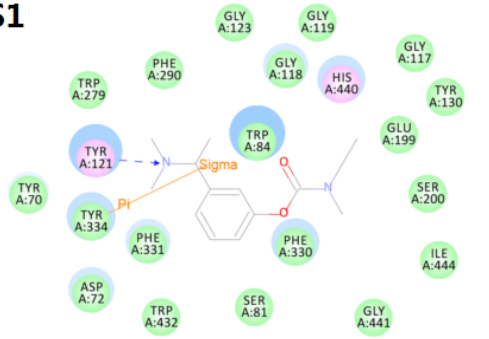

R1

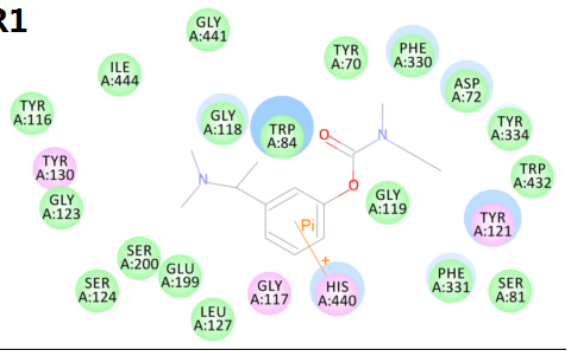

R2

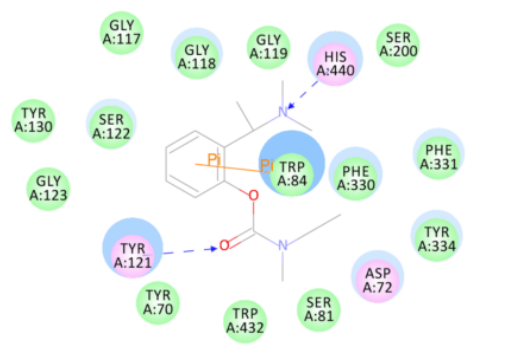

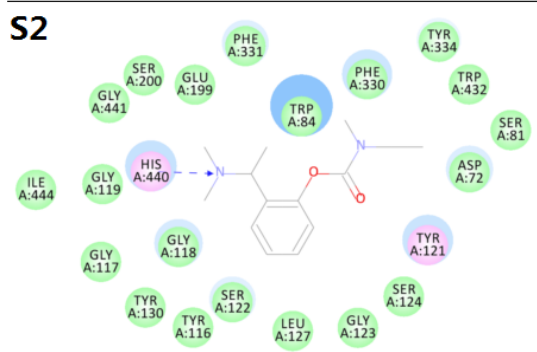

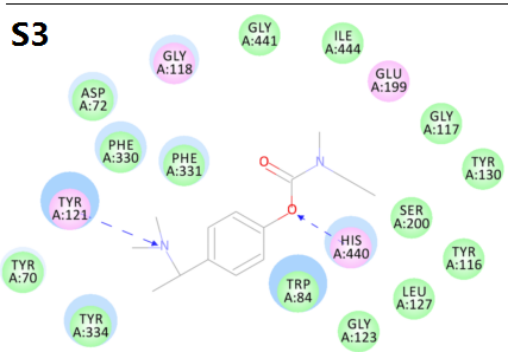

R3

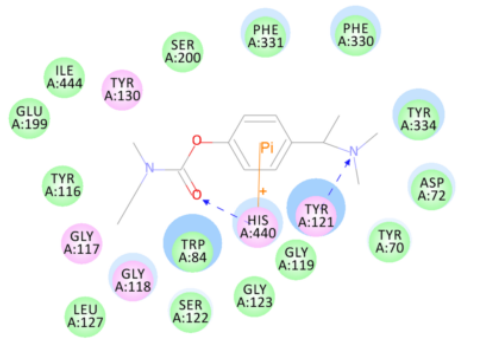

Figure 3. The 2D binding patterns of the studied compounds into the active site of $\mathrm{AChE}$

the molecular docking simulation. Thus, the future modification can be cautiously carried out consulting these key interactions.

\section{Conclusion}

In summary, more possibilities on substituent-positions and conformations from Rivastigmine were revealed. The molecular docking indicated that the para-position might be the orientation of future modification and the S-conformation should be retained. The key interactions with residues including TYR121 and HIS440 should be treated with extra caution in the future design plans. The information raised in this work might lead to further investigation of AChEIs and even corresponding therapeutic approaches in treating Alzheimer's disease.

\section{References}

1. van Der Berg N, Beekman M, Smith KR, Janssens A, Slagboom PE (2017) Historical demography and longevity genetics: Back to the future. Ageing Res Rev 38: 28-39. [Crossref]

2. Morley JE, Farr SA, Nguyen AD (2018) Alzheimer Disease. Clin Geriatr Med 34: 591-601. [Crossref]

3. de Waal H, Stam CJ, de Haan W, Van Straaten EC, Scheltens P, et al. (2012) Young Alzheimer patients show distinct regional changes of oscillatory brain dynamics. Neurobiol Aging 33: 1008e25-1008e31. [Crossref]

4. Burns A, Iliffe S (2009) Alzheimer's disease. BMJ 338: b1349. [Crossref]

5. Ballard C, Gauthier S, Corbett A, Brayne C, Aarsland D, et al. (2011) Alzheimer's disease. Lancet 377: 1019-1031. [Crossref]

6. Alzheimer's Association (2017) 2017 Alzheimer's disease facts and figures. Alzheimer's \& Dementia 13: 325-373. [Crossref]

7. Alzheimer's Association (2018) 2018 Alzheimer's disease facts and figures. Alzheimer's \& Dementia 14: 367-429.
8. Pu Z, Ma S, Wang L, Li M, Shang L, et al. (2018) Amyloid-beta degradation and neuroprotection of Dauricine mediated by unfolded protein response in a Caenorhabditis elegans model of Alzheimer's disease. Neuroscience 392: 25-37. [Crossref]

9. Jembrek MJ, Slade N, Hof PR, Simic G (2018) The interactions of p53 with tau and $A \beta$ as potential therapeutic targets for Alzheimer's disease. Prog Neurobiol 168: 104127. [Crossref]

10. Koppel J, Acker C, Davies P, Lopez OL, Jimenez H, et al. (2014) Psychotic Alzheimer's disease is associated with gender-specific tau phosphorylation abnormalities. Neurobiol Aging 35: 2021-2028. [Crossref]

11. Selkoe DJ (1999) Translating cell biology into therapeutic advances in Alzheimer's disease. Nature 399: A23-A31. [Crossref]

12. Francis PT, Palmer AM, Snape M, Wilcock GK (1999) The cholinergic hypothesis of Alzheimer's disease: a review of progress. J Neurol Neurosurg Psychiatry 66: 137-147. [Crossref]

13. Martorana A, Esposito Z, Koch G (2010) Beyond the cholinergic hypothesis: do current drugs work in Alzheimer's disease? CNS Neurosci Ther 16: 235-245. [Crossref]

14. Gu ZL, Alexander GM, Dudek SM, Yakel JL (2017) Hippocampus and entorhina cortex recruit cholinergic and NMDA receptors separately to generate hippocampal theta oscillations. Cell Rep 21: 3585-3595. [Crossref]

15. Hardy J, Allsop D (1991) Amyloid deposition as the central event in the aetiology of Alzheimer's disease. Trends Pharmacol Sci 12: 383-388. [Crossref]

16. Nistor M, Don M, Parekh M, Sarsoza F, Goodus M, et al. (2007) Alpha- and betasecretase activity as a function of age and beta-amyloid in Down syndrome and normal brain. Neurobiol Aging 28: 1493-1506. [Crossref]

17. Polvikoski T, Sulkava R, Haltia M, Kainulainen K, Vuorio A, et al. (1995) Apolipoprotein E, dementia, and cortical deposition of $\beta$-amyloid protein. $N$ Eng J Med 333: 1242-1248. [Crossref]

18. Lauren J, Gimbel DA, Nygaard HB, Gilbert JW, Strittmatter SM (2009) Cellular prion protein mediates impairment of synaptic plasticity by amyloid- $\beta$ oligomers. Nature 457: 1128-1132. [Crossref]

19. Goedert M, Spillantini MG, Crowther RA (1991) Tau proteins and neurofibrillary degeneration. Brain Pathol 1: 279-286. [Crossref] 
20. Iqbal K, Alonso AC, Chen S, Chohan MO, El-Akkad E, et al. (2005) Tau pathology in Alzheimer disease and other tauopathies. Biochim Biophys Acta 1739: 198-210. [Crossref]

21. Chun WJ, Johnson GVW (2007) The role of tau phosphorylation and cleavage in neuronal cell death. Front Biosci 12: 733-756. [Crossref]

22. Deane R, Zolkovic BV (2007) Role of the blood-brain barrier in the pathogenesis of Alzheimer's disease. Curr Alzheimer Res 4: 191-197. [Crossref]

23. Su B, Wang XL, Nunomura A, Moreira PI, Lee HG, et al. (2008) Oxidative stress signaling in Alzheimer's disease. Curr Alzheimer Res 5: 525-532. [Crossref]

24. Bondy SC (2016) Low levels of aluminum can lead to behavioral and morphological changes associated with Alzheimer's disease and age-related neurodegeneration. Neurotoxicology 52: 222-229. [Crossref]

25. Kandimalla R, Vallamkondu J, Corgiat EB, Gill KD (2016) Understanding aspects of Aluminum exposure in Alzheimer's disease development. Brain Pathol 26: 139-154. [Crossref]
26. Qiang L, Sun XH, Austin TO, Muralidharan H, Jean DC, et al. (2018) Tau does not stabilize axonal microtubules but rather enables them to have long labile domains. Curr Biol 28: 2181-2189. [Crossref]

27. Qizilbash N, Whitehead A, Higgins J, Wilcock G, Schneider L, et al. (1998) Cholinesterase inhibition for Alzheimer disease: A meta-analysis of the Tacrine trials. JAMA 280: 1777-1782. [Crossref]

28. Rodrigues Simoes MC, Dias Viegas FP, Moreira MS, De Freitas Silva M, Riquiel MM, et al. (2014) Donepezil: an important prototype to the design of new drug candidates for Alzheimer's disease. Mini Rev Med Chem 14: 2-19. [Crossref]

29. Heinrich M, Teoh HL (2004) Galanthamine from snowdrop - the development of a modern drug against Alzheimer's disease from local Caucasian knowledge. $J$ Ethnopharmacol 92: 147-162. [Crossref]

30. Burn D, Emre M, McKeith I, De Deyn PP, Aarsland D, et al. (2006) Effects of rivastigmine in patients with and without visual hallucinations in dementia associated with Parkinson's disease. Movement Disord 21: 1899-1907. [Crossref]

Copyright: $@ 2019$ Sun Z. This is an open-access article distributed under the terms of the Creative Commons Attribution License, which permits unrestricted use, distribution, and reproduction in any medium, provided the original author and source are credited. 